\title{
FRICKE'S OCTAVIC CURVE
}

\author{
by W. L. EDGE
}

(Received 31st May, 1983)

\section{Summary}

The paper is concerned with a curve $F$, the complete intersection of a quadric with a quartic surface, that admits a group of self-projectivities isomorphic to the symmetric group of degree 5. Every generator of the quadric is, as shown at the end of the paper, cut by $F$ equianharmonically. $F$ has 80 stalls, points where its osculating plane is stationary; they are of two kinds, 60 to be labelled $\Sigma$, the other $20 \Omega$. $F$ also has inflections at 24 points which compose a figure encountered on earlier occasions. A search is made for tritangent planes of $F$ of which, when reckoned according to proper multiplicity, there must be 2048. Among them are 60 all of whose three contacts are $\Sigma$ while a further 120 each involve a single $\Sigma$ among their contacts and 420 each involve a single $\Omega$.

The existence of the group of self-projectivities is due to the presence of a certain basic pentahedron, so that the paper opens by describing this.

\section{Preamble}

Some account has been given $[3,4]$, using a system of supernumerary homogeneous coordinates $x, y, z, t, u$ subject to the identity

$$
S_{1} \equiv x+y+z+t+u=0
$$

of the geometry of Bring's sextic curve $B$, the intersection of the quadric

$$
Q: S_{2} \equiv x^{2}+y^{2}+z^{2}+t^{2}+u^{2}=0
$$

and the "diagonal" cubic surface

$$
D: S_{3} \equiv x^{3}+y^{3}+z^{3}+t^{3}+u^{3}=0 .
$$

It is now proposed to study, analogously, the intersection $F$ of $Q$ with the quartic surface

$$
\Phi: S_{4} \equiv x^{4}+y^{4}+z^{4}+t^{4}+u^{4}=0
$$

$F$ is an octavic curve, quadrisecant to every generator of $Q$. Since its plane projection 
from a point of itself has order 7 and has no multiple points other than two triple points [2] it has genus 9 .

It seems proper to say, notwithstanding the fleeting reference [7, p. 202] to the curve $\beta=0$, that $F$ is Fricke's octavic. He not only explicitly mentions [5, p. 368] this irreducible curve: he gives its genus and emphasises its invariance under a group of 120 self-transformations; but although he notes that invariant sets of 20,24 points are intersections of $F$ with certain special surfaces he does not give the particular geometrical attributes that serve to identify them and another set of 60 , and which it is intended to give below $(\$ \$ 2,3,4)$.

The faces of the pentahedron of reference $P$ meet by pairs in ten edges $e_{i j}$, by threes in ten vertices $V_{i j} ; V_{i j}$ is opposite to the edge common to those two faces not containing $V_{i j}$ and is joined to it by the diagonal plane $d_{i j}$. Examples are

$$
V_{12}:(1,-1,0,0,0) ; \quad e_{12}: x=y=0=z+t+u ; \quad d_{12}: x+y=0=z+t+u .
$$

Of course either, at choice, of the two equations given suffices to determine $d_{12}$, and any two of the three linearly dependent equations determine $e_{12}$.

The join of two vertices whose binary suffixes are disjoint is a diagonal; there are fifteen of them; the three through $V_{i j}$ join it to the vertices on $e_{i j}$. One diagonal joins

$$
V_{23}(0,1,-1,0,0) \text { and } V_{45}(0,0,0,1,-1) \text {, }
$$

its equations being $x=y+z=t+u=0$. All the diagonals are on $D$, which is therefore called the diagonal surface, as indeed they are on all the surfaces $S_{2 n+1}=0$.

The polar plane $\pi_{i j}$ of $V_{i j}$ with respect to $Q$ contains $e_{i j}$; that of $V_{12}$ is $\pi_{12}: x=y$. The product of the ten $\pi_{i j}$ is Fricke's $A_{10}$.

\section{Twenty points $\Omega$}

Each $d_{i j}$ meets $\Phi$ in a pair of conics with double contact: the points common to $\Phi$ and $d_{12}$ satisfy

$$
\begin{gathered}
x^{4}+x^{4}+z^{4}+t^{4}+(z+t)^{4}=0, \\
x^{4}+\left(z^{2}+z t+t^{2}\right)^{2}=0, \\
\left(z^{2}+z t+t^{2}+i x^{2}\right)\left(z^{2}+z t+t^{2}-i x^{2}\right)=0,
\end{gathered}
$$

a pair of conics in $d_{12}$ both touching the lines $z=\omega t, z=\omega^{2} t$ where they meet $x=0$, the two tangents meeting at $V_{12}$; here, as customary, $\omega$ is a complex cube root of 1 .

So ten among the plane sections of $\Phi$ are pairs of conics with double contact. These contacts are on edges of $P$, those on $e_{12}$ being $\left(0,0,1, \omega, \omega^{2}\right)$ and $\left(0,0,1, \omega^{2}, \omega\right)$ which are the intersections of $e_{12}$ with $Q$ : the edges of $P$ are bitangents of $\Phi$ and chords of $F$. Their 20 intersections with $F$ are geometrically significant: call them $\Omega$-points. 
Since the tangent of $F$ at $\left(0,0,1, \omega, \omega^{2}\right)$ is

$$
z+\omega t+\omega^{2} u=z+t+u=0
$$

common to the tangent planes of $Q$ and $\Phi$ it contains $V_{12}$. The passage of the tangents of $F$ at both $\Omega$ on $e_{i j}$ through $V_{i j}$ involves tritangent planes of $F$ : for example, $\omega u=\omega^{2} t$ through $e_{45}$ contains

$$
\begin{aligned}
& \text { the join of } V_{12} \text { on } e_{45} \text { to }\left(0,0,1, \omega, \omega^{2}\right) \text { on } e_{12} \text {, } \\
& \text { the join of } V_{23} \text { on } e_{45} \text { to }\left(1,0,0, \omega, \omega^{2}\right) \text { on } e_{23} \text {, } \\
& \text { the join of } V_{31} \text { on } e_{45} \text { to }\left(0,1,0, \omega, \omega^{2}\right) \text { on } e_{31} \text {. }
\end{aligned}
$$

All of these joins are tangents of $F$, so that $u=\omega t$ is a tritangent plane (and meets $F$ further in the $\Omega$ on $e_{45}$ ). So one finds 20 tritangent planes, two through each $e_{i j}$.

Note that $d_{12}$ meets $Q$ in the conic $x^{2}+z^{2}+z t+t^{2}=0$ which belongs to the pencil containing the two conics on $\Phi$. Hence, as $F$ is the common curve of $Q$ and $\Phi, d_{12}$ has four-point intersection with $F$ at both points of the $\Omega$ pair. All $20 \Omega$ are stalls on $F$ and $d_{12}$ the stationary osculating plane at both.

\section{Twenty-four points $I$}

On p. 542 of [3] there were noticed 24 points of $B$ that happen to be also on $F$; they are the intersections of $B$ with $\Phi$ and compose the complete set of points common to the three surfaces $Q, D, \Phi$. They are obtained from

$$
a\left(1, \varepsilon, \varepsilon^{2}, \varepsilon^{3}, \varepsilon^{4}\right)
$$

by imposing all 4 ! permutations on its $y, z, t, u$ coordinates; here $\varepsilon$ is $\exp (2 \pi i / 5)$. Certain pairs of these points are conjugate with respect to $Q$, so that their joins are generators. Any point

$$
\left(1, \varepsilon^{p}, \varepsilon^{q}, \varepsilon^{r}, \varepsilon^{s}\right)
$$

conjugate to $a$ is in the tangent plane

$$
x+\varepsilon y+\varepsilon^{2} z+\varepsilon^{3} t+\varepsilon^{4} u=0
$$

to $Q$ and so

$$
1+\varepsilon^{p+1}+\varepsilon^{q+2}+\varepsilon^{r+3}+\varepsilon^{s+4}=0
$$

which will hold if (like $p, q, r, s) p+1, q+2, r+3, s+4$ are a permutation $(\bmod 5)$ of 1 , 2,3 , 4. Thus $p, q+1, r+2, s+3$ are, in some order, $0,1,2,3$ and, as $p \neq 0$, there are only 
three disjoint possibilities:

$$
q=4, \quad r=3, \quad s=2 .
$$

Discussion is similar in all three instances. If, for example, $q=4$ then $p, r, s$ are 1, 2, 3 as also are $p, r+2, s+3$; so consecutively, $s=3, r=1, p=2$ and $a$ is conjugate to $b$ $\left(1, \varepsilon^{2}, \varepsilon^{4}, \varepsilon, \varepsilon^{3}\right)$. If $r=3$ one returns to a which is of course conjugate to itself; if $s=2$ one arrives at $\bar{b}\left(1, \varepsilon^{3}, \varepsilon, \varepsilon^{4}, \varepsilon^{2}\right)$. As $b, \bar{b}$ are transposed by complex conjugation they are both conjugate to $\bar{a}\left(1, \varepsilon^{4}, \varepsilon^{3}, \varepsilon^{2}, \varepsilon\right)$. Thus there is on $Q$ a quadrilateral whose vertices belong to both $B$ and $F$ and whose sides are generators, two in each regulus, and whose diagonals $a \bar{a}, b \bar{b}$ are polar lines.

$$
\begin{array}{ll}
a\left(1, \varepsilon, \varepsilon^{2}, \varepsilon^{3}, \varepsilon^{4}\right) & \bar{b}\left(1, \varepsilon^{3}, \varepsilon, \varepsilon^{4}, \varepsilon^{2}\right) \\
b\left(1, \varepsilon^{2}, \varepsilon^{4}, \varepsilon, \varepsilon^{3}\right) & \bar{a}\left(1, \varepsilon^{4}, \varepsilon^{3}, \varepsilon^{2}, \varepsilon\right) .
\end{array}
$$

There are six such quadrilaterals: they arise from the one here displayed by keeping $x$ and one of the other coordinates fixed while imposing the 3 ! permutations on the remaining three.

It was explained $[3$, p. 543$]$ that the generators

$$
a b, b \bar{a}, \bar{a} \bar{b}, \bar{b} a
$$

are the tangents of $B$ at

$$
a, b, \bar{a}, \bar{b} .
$$

They will now be seen to be the tangents of $F$ at

$$
b, \bar{a}, \bar{b}, a
$$

indeed not only do they touch $F$, they are inflectional tangents. For the coordinates of points on, say, $a b$ occur on varying $\rho, \sigma$ in

$$
\rho+\sigma, \quad \rho \varepsilon+\sigma \varepsilon^{2}, \quad \rho \varepsilon^{2}+\sigma \varepsilon^{4}, \quad \rho \varepsilon^{3}+\sigma \varepsilon, \quad \rho \varepsilon^{4}+\sigma \varepsilon^{3}
$$

whose sum (of course) is zero, as the sum of their squares is too. But their cubes sum to $15 \rho \sigma^{2}$, their fourth powers to $20 \rho^{3} \sigma$ so that the three intersections of $B$ and $a b$ consist of one at $b$ and two at $a$ while the four intersections of $F$ and $a b$ consist of three at $b$ and one at $a$.

Call these inflections of $F$ the 24 points $I$. The same argument as was used [3, p. 543] to identify osculating planes of $B$ shows, when applied to $F$, that the planes having 4point intersection with $F$ at $a, b, \bar{a}, \bar{b}$ are the respective tangent planes $\bar{b} a b, a b \bar{a}, b \bar{a} \bar{b}, \bar{a} \bar{b} a$ of $Q$. So far as their relation to $B$ is concerned the six quadrilaterals were detected by Wiman [10, p. 19]; he also identified [10, p. 20] the osculating planes of $B$ at their 24 vertices. 
Take now the chord $c d$ of $F$ to be a side of any of these six quadrilaterals; every plane through $c d$ has three-point intersection with $F$ at either $c$ or $d$. Those ten planes which join $c d$ to lines $\lambda$ in the opposite regulus belonging to those five quadrilaterals not involving $c d$ each have three-point intersection with $F$ also at one of the two intersections of $\lambda$ with $F$; thus 60 tangent planes of $Q$ have three-point intersections with $F$ at two distinct places. These are not "ordinary" double osculating planes: they are specialised in the sense that their three coincident intersections at either contact are collinear.

If, however, $\lambda$ belongs to the same quadrilateral as $c d$ and so contains either $c$ or $d$ one is concerned with the intersections of $F$ with the tangent plane of $Q$ at a point $I$. Take $a$, with tangent plane $\bar{b} a b$; the points of this plane are obtained by varying $\rho, \sigma, \tau$ in $\rho+\sigma+\tau, \rho \varepsilon+\sigma \varepsilon^{2}+\tau \varepsilon^{3}, \rho \varepsilon^{2}+\sigma \varepsilon^{4}+\tau \varepsilon, \rho \varepsilon^{3}+\sigma \varepsilon+\tau \varepsilon^{4}, \rho \varepsilon^{4}+\sigma \varepsilon^{3}+\tau \varepsilon^{2}$ for which

$$
S_{2}=10 \sigma \tau, \quad S_{4}=10\left(2 \rho^{3} \sigma+2 \rho \tau^{3}+3 \sigma^{2} \tau^{2}\right),
$$

so that the eight points of $F$ occur when

$$
\sigma=0=\rho \tau^{3} \quad \text { and } \quad \tau=0=\rho^{3} \sigma .
$$

The former set is $b+3 a$, the latter is $a+3 b$ so that the complete intersection is $b+4 a$ $+3 b$. This plane is clearly a tritangent plane two of whose three contacts coalesce at $a$; but it is further specialised in that its third contact is not an ordinary contact but a three-point contact, and this latter specialisation entitles it to be reckoned twice. So the tangent planes of $Q$ at the $24 I$ contribute 48 to the tally of tritangent planes of $F$.

Just as the intersection of $F$ with the tangent plane of $Q$ at $a$ consists of $5+4 a+3 b$ its intersection with the tangent plane of $Q$ at $\bar{a}$ likewise consists of $b+4 \bar{a}+3 \bar{b}$; so the pair of planes cuts $F$ in the set $4(a+b+\bar{a}+\bar{b})$. This same quadruple tetrad is also cut on $F$ by the pair of planes tangent to $Q$ at $b$ and $b$. Thus every quadric, other than $Q$ itself, belonging to the pencil whose base is the skew quadrilateral $a b \bar{a} \bar{b}$ cuts this special set on $F$. This is the more significant in that the canonical sets on $F$ happen to be its intersections with quadrics, so that while the canonical sets on any curve involve some every one of whose points is reckoned twice-in general there is a finite number $2^{p-1}\left(2^{p}\right.$ $-1)$ of these double sets on a curve of genus $p-F$ is so specialised that six of its canonical sets consist of four points each reckoned four times.

\section{Sixty points $\Sigma$}

The chief feature of $F$, as of $B$, is invariance under the 120 operations of the group $S_{5}$ of permutations of the coordinates. The least complicated permutations are transpositions, leaving three coordinates unchanged while transposing the residual pair. Here there are ten of them; each is achieved by a harmonic inversion $h_{i j}$ in a vertex $V_{i j}$ of $P$ and its polar plane $\pi_{i j}$ with respect to $Q$. Take, for instance, $V_{12}(1,-1,0,0,0)$; its polar plane $\pi_{12}$ is $x=y$ and

$$
(x, y, z, t, u) \quad(y, x, z, t, u)
$$


are transposed by $h_{12}$ because the difference of these coordinate vectors gives $V_{12}$, their sum a point in $\pi_{12}$. Each point of $F$ is joined to $V_{12}$ by a chord of $F$, the two intersections with $F$ being images in $h_{12}$. But should the point be among the eight intersections of $F$ with $\pi_{12}$ the tangent of $F$ passes through $V_{12}$ and an earlier discussion [3, p. 541] shows all these points to be stalls on $F$ where its osculating plane is stationary, having four-point intersection. It also shows this stationary osculating plane to be the tangent plane of the cone of chords. Two of these eight stalls are the $\Omega$ on $e_{12}$; call the other six points $\Sigma$. In this way

$$
20+6.10=80
$$

stalls of $F$ are accounted for, and there are no others. For the planes of space cut $F$ in the octads of a $g_{8}^{3}$ and a standard formula [9, p. 188] gives $(3+1)(8+3.9-3)=128$ for the number of octads with a quadruple member. But these certainly include the 24 points $I$, and indeed twice over. This multiplicity is also a standard matter; an inflection, while counting singly in every octad cut by a plane through it but not containing the tangent, counts trebly among all those planes which do pass through the tangent, quadruply in a unique plane, so that the appropriate multiplicity is $[8, \mathrm{p} .77$; or, for a text book reference, 9, p. 188]

$$
1+3+4-\frac{1}{2} \cdot 3 \cdot 4=2
$$

So $128-48=80$ is the tally of stalls on $F$. Each plane $\pi_{i j}$ meets $F$ at eight stalls, two of them $\Omega$, the other six $\Sigma$.

\section{Ten quartic cones of chords}

The eight intersections of a plane through $V_{12}$ with $F$ consist of four pairs: each member of a pair is the image of the other in $h_{12}$; in other words, the chords of $F$ through $V_{12}$ generate a quartic cone $q_{12}$. Among its generators are $e_{34}, e_{35}, e_{45}$ each joining a pair $\Omega$, and the tangent plane of $q_{12}$ along, say, $e_{34}$ contains the tangents of $F$ at both members of the $\Omega$-pair; these tangents, as was implied in $\S 2$, meet at $V_{34}$. But the plane is $d_{34}$, having four-point intersection with $F$ at both the $\Omega$, so that the tangent plane of $q_{12}$ along $e_{34}$ meets $q_{12}$ only in $e_{34}$ reckoned four times. The section of $q_{12}$ by a plane not containing $V_{12}$ is a quartic curve, non-singular as will be seen, with undulations on $e_{34}, e_{35}, e_{45}$.

The equation of $q_{12}$ is the outcome of eliminating $x$ and $y$ from (1.1), (1.2) and (1.3), and the identity

$$
(x+y)^{4}+2\left(x^{4}+y^{4}\right) \equiv\left(x^{2}+y^{2}\right)\left\{x^{2}+y^{2}+2(x+y)^{2}\right\}
$$

shows the outcome to be

$$
(z+t+u)^{4}-2\left(z^{4}+t^{4}+u^{4}\right)=\left(z^{2}+t^{2}+u^{2}\right)\left\{z^{2}+t^{2}+u^{2}-2(z+t+u)^{2}\right\}
$$

wherein fourth powers cancel in accordance with $e_{34}, e_{35}, e_{45}$ being on $q_{12}$. The 
undulations are apparent on writing (5.1) as

$$
(t+u)(u+z)(z+t)(z+t+u)=(t u+u z+z t)^{2}
$$

showing $t+u=0$ to have all its four intersections with this plane quartic coincident at $t=u=0$. The bitangency of $z+t+u=0$ (the section of $d_{12}$ ) and its points $\Omega$ of contact are also apparent, and the curve is easily shown to be free of multiple points.

So $F$ is, in ten different ways, in $(2,1)$ correspondence with a curve of genus 3 . And this curve is in itself special being, as the symmetry of (5.2) shows, invariant under a dihedral group of six self-projectivities. It has three undulations, each accounting for two of its 24 inflections because while its Hessian sextic cuts it at its inflections it touches it at any undulation. The equation (5.2) shows that its three undulation tangents touch a conic at their contacts, and that the Hessian duad on the conic of these three contacts are contacts of the quartic with a bitangent.

Apart from the three edges of $P$ through $V_{i j}$, undulation generators of $q_{i j}$, the cone has $24-2.3=18$ inflectional generators; its tangent planes along these are double osculating planes of $F$, the two contacts of each being paired in $h_{i j}$. So 180 such planes are recognised.

6.

The 28 bitangent planes each touching a quartic cone along two of its generators include, for a cone $q_{i j}$, diagonal planes with special properties. For example: in $d_{34}, d_{35}$, $d_{45}$ the two generators of contact with $q_{12}$ coincide on an undulation generator, so that 25 bitangent planes remain; these include $d_{12}$ which does touch $q_{12}$ along distinct generators, but is special in that the two contacts of $F$ with $d_{12}$ on either coincide at an $\Omega$ on $e_{12}$.

There are, apart from diagonal planes, 24 bitangent planes of each $q_{i j}$; they are quadritangent to $F$ so that each is to be counted as four tritangent planes and one obtains a contribution of 960 to the full number of tritangent planes of $F$.

\section{Tritangent planes whose three contacts are $\Sigma$}

Tritangent planes all of whose three contacts with $F$ are stalls occur just as with $B$ [4, p. 216], though of course with $B$ there were no additional intersections. If $(1,1, \xi, \eta, \zeta)$ is a $\Sigma$ in $\pi_{12}$

$$
\xi+\eta+\zeta=\xi^{2}+\eta^{2}+\zeta^{2}=\xi^{4}+\eta^{4}+\zeta^{4}=-2
$$

so that $\xi, \eta, \zeta$ are the zeros of

$$
\theta^{3}+2 \theta^{2}+3 \theta+\frac{3}{2}
$$

The six $\Sigma$ in $\pi_{12}$ occur on permuting $\xi, \eta, \zeta$, no two of them being equal; all $60 \Sigma$ occur on permuting $1,1, \xi, \eta, \zeta$ in all $5 ! / 2 !=60$ possible different ways. The generator

$$
z / \xi=t / \eta=u / \zeta
$$


of $q_{12}$ touches $F$ at $(1,1, \xi, \eta, \zeta)$ and the plane $t / \eta=u / \zeta$ joining (7.2) to $e_{45}$ is tritangent to $F$ at

$$
(\xi, 1,1, \eta, \zeta), \quad(1, \xi, 1, \eta, \zeta), \quad(1,1, \xi, \eta, \zeta)
$$

In $\pi_{12}$, correspondingly, the join $t / \eta=u / \zeta$ of $(\xi, \eta, \zeta)$ on (5.2) to the undulation $(1,0,0)$ touches $(5.2)$ at $(1, \eta, \zeta)$; this can be directly verified, using the fact that (7.1) is zero when $\theta=\xi, \eta, \zeta$.

So each $q_{i j}$ has six generators, each belonging to three tritangent planes whose contacts are all $\Sigma$, and each such plane so arises from three $q_{i j}$. Thus 60 tritangent planes of $F$ are identified.

\section{Further tritangent planes}

Now, again as in [4, p. 216], there are, through these 60 generators of the $q_{i j}$ tritangent planes only one of whose contacts is a stall. For through any point other than an inflection on a non-singular plane quartic there pass ten lines touching the curve elsewhere, so that through a join of $V_{i j}$ to a point $\Sigma$ in $\pi_{i j}$ there pass ten planes touching $q_{i j}$ along other generators $g$. But among these are three of the tritangent planes of $F$ just noticed; seven other planes remain which are tritangent to $F$, their other two contacts being images on $g$ in $h_{i j}$. Each $q_{i j}$ thus affords 42 such planes, so that 420 more of the tritangent planes of $F$ are found.

There are also ten planes through the join of $V_{i j}$ to either $\Omega$ on $e_{i j}$ that touch $q_{i j}$ along other generators; $d_{i j}$ is one of these, and they also include three of those noted in $\S 2$. For example: $\left(0,0,1, \omega, \omega^{2}\right)$ is joined to $V_{12}$ by the line $z=\omega^{2} t=\omega u$ lying in the three planes

$$
\omega^{2} t=\omega u, \quad \omega^{2} u=\omega z, \quad \omega^{2} z=\omega t
$$

meeting $\pi_{12}$ in the tangents of (5.2) at

$$
\left(0, \omega, \omega^{2}\right), \quad\left(\omega^{2}, 0, \omega\right), \quad\left(\omega, \omega^{2}, 0\right)
$$

There remain, apart from $z+t+u=0$, six further tangents of (5.2) through $\left(0,0,1, \omega, \omega^{2}\right)$; their joins to $V_{12}$ are tritangent planes of $F$, and the companion $\Omega$ on $e_{12}$ provides another six. So, in all, 120 more tritangent planes of $F$ are found.

9.

Apart from the ten planes $d_{i j}$ the number of tritangent planes accounted for is

$$
20+48+60+960+420+120=1628
$$

and to these must be added the $d_{i j}$ counted with their proper multiplicity. Any tritangent plane not fixed by any of the 120 self-projectivities of $F$ will belong to an orbit of 120 . 
10.

Among the many equations, obtained by Cayley $[1$, p. 77$]$, involving the characters of an algebraic curve $C$ in [3] is the pair, both self-dual,

$$
\begin{aligned}
& t+t^{\prime}=\frac{1}{3}(r-4)(r-5)(r-6)-4 p(r-10)-i(r-6)-2 \tau(r-8) \\
& t-t^{\prime}=(r-6)(n-m)
\end{aligned}
$$

where $t$ is the number of tritangent planes, $t^{\prime}$ the number of triads of concurrent tangents. Here $n$ is the order of $C, m$ the class (number of osculating planes through a point), $r$ the rank (number of tangents meeting a line), $p$ the genus, $i$ the number of inflections, $\tau$ the number of double tangents. For $F$

$$
n=8, \quad m=48, \quad r=32, \quad p=9, \quad i=24, \quad \tau=0,
$$

so that $t=2048, t^{\prime}=3088$. If it could convincingly be shown that each $d_{i j}$ accounts for six tritangent planes one could observe that

$$
2048=1688+360
$$

allowing for three orbits of 120 .

\section{The equianharmonic property}

Since the four points $a, b, \bar{a}, \bar{a}$ of (3.1) are linearly independent any point in the space has, for some $p, q, r, s$ coordinates given by

$$
(x, y, z, t, u)=(p, q, r, s)\left(\begin{array}{ccccc}
1 & \varepsilon & \varepsilon^{2} & \varepsilon^{3} & \varepsilon^{4} \\
1 & \varepsilon^{2} & \varepsilon^{4} & \varepsilon & \varepsilon^{3} \\
1 & \varepsilon^{3} & \varepsilon & \varepsilon^{4} & \varepsilon^{2} \\
1 & \varepsilon^{4} & \varepsilon^{3} & \varepsilon^{2} & \varepsilon
\end{array}\right)
$$

this is to supersede the supernumerary coordinates by homogeneous coordinates with $a b \bar{b} \bar{a}$ as tetrahedron of reference. Then [7, p. 187]

$$
\frac{1}{10} \Sigma x^{2}=p s+q r, \quad \frac{1}{10} \Sigma x^{4}=2\left(p^{3} q+q^{3} s+s^{3} r+r^{3} p\right)+3\left(p^{2} s^{2}+q^{2} r^{2}\right)+12 p q r s
$$

Now parametrise $Q$, with equation $p s+q r=0$, by

$$
p: q: r: s=\lambda_{1} \mu_{1}: \lambda_{2} \mu_{1}: \lambda_{1} \mu_{2}:-\lambda_{2} \mu_{2}
$$

so that $[7$, pp. 179,180$] \lambda_{1} / \lambda_{2}$ and $\mu_{1} / \mu_{2}$ are parameters in its two reguli. This point satisfies $\Sigma x^{4}=0$, and so lies on $F$ if, and only if $[6$, p. 381; 7, p. $196 ; 5$, p. 368]

$$
\lambda_{1}^{4} \mu_{1} \mu_{2}^{3}+\lambda_{1}^{3} \lambda_{2} \mu_{1}^{4}-3 \lambda_{1}^{2} \lambda_{2}^{2} \mu_{1}^{2} \mu_{2}^{2}-\lambda_{1} \lambda_{2}^{3} \mu_{2}^{4}-\lambda_{2}^{4} \mu_{1}^{3} \mu_{2}=0
$$


the double binary quartic accords with $F$ being quadrisecant to every line on $Q$.

A binary quartic

$$
a X^{4}+4 b X^{3} Y+6 c X^{2} Y^{2}+4 d X Y^{3}+e Y^{4}
$$

has two invariants

$$
I \equiv a e-4 b d+3 c^{2}, \quad J \equiv\left|\begin{array}{lll}
a & b & c \\
b & c & d \\
c & d & e
\end{array}\right|,
$$

and discriminant $\Delta \equiv I^{3}-27 J^{2}$. But, regarding (11.1) as a binary quartic in $\lambda_{1}, \lambda_{2}$ so that

$$
a=\mu_{1} \mu_{2}^{3}, \quad b=\frac{1}{4} \mu_{1}^{4}, \quad c=-\frac{1}{2} \mu_{1}^{2} \mu_{2}^{2}, \quad d=-\frac{1}{4} \mu_{2}^{4}, \quad e=-\mu_{1}^{3} \mu_{2},
$$

$I$ is identically zero, as it is also, similarly, for (11.1) regarded as a binary quartic in $\mu_{1}, \mu_{2}$. Thus every line on $Q$ is cut equianharmonically by $F$.

Incidentally: whenever $I=0 \Delta$ is a multiple of $J^{2}$; this accords with $F$ having not an ordinary contact but an inflection whenever it touches a line on $Q$. A last point of interest is that (11.2) make

$$
J=\frac{1}{16} \mu_{1} \mu_{2}\left(\mu_{1}^{10}+11 \mu_{1}^{5} \mu_{2}^{5}-\mu_{2}^{10}\right) ;
$$

the parameters of the twelve lines in the " $\mu$ " regulus that are inflectional tangents of $F$ are zeros of this $[7$, p. 56] icosahedral duodecimic; and the same is true of the " $\lambda$ " regulus.

\section{REFERENCES}

1. A. CAYley, On the theory of the curve and torse, Quarterly J. of Pure and Applied Maths. 11 (1871), 294-317: Mathematical Papers 8 (Cambridge Univ. Press, 1895), 72-91.

2. A. CAyley, On the curves situate on a surface of the second order, Phil. Mag. 22 (1861), 35 38: Mathematical Papers 5 (Cambridge Univ. Press, 1892), 70-72.

3. W. L. EdGe, Bring's curve, J. London Math. Soc. (2) 18 (1978), 539-545. 222.

4. W. L. EdGe, Tritangent planes of Bring's curve, J. London Math. Soc. (2) 23 (1981), 215-

5. R. FrICKe, Entwicklungen zur Transformationen fünfter und siebenter Ordnung einiger spezieller automorpher Funktionen, Acta Mathematica 17 (1893).

6. P. Gordan, Ueber die Auflösung der Gleichungen von fünften Grade, Math. Annalen 13 (1878), 375-404.

7. F. KLeiN, Vorlesungen über das Ikosaeder (Teubner, Leipzig, 1884).

8. C. Segre, Introduzione alla geometria sopra un ente algebrico semplicemente infinito, Annali di mat. (2) 22 (1894), 41-142: Opere I (Rome, 1957), 198-304. 
9. F. SEveri, Vorlesungen über algebraische Geometrie (Teubner, Leipzig, 1921).

10. A. Wiman, Ueber die algebraischen Curven von den Geschlechtern $p=4,5$, 6 welche eindeutige Transformationen in sich besitzen, Svenska Vet. Akad. Handlingar: Bihang till Handlingar 21 (1895), afd. 1, No. 3, 41 pp.

INVERESK HOUSE

MuSSELBURGH

EH21 7UA 